D.T. Wickramasinghe, L. Ferrario, and G.V. Bicknell, eds.

\title{
Inside the Bullets of Orion
}

\author{
Michael G. Burton \\ School of Physics, University of New South Wales, Sydney, NSW 2052, \\ Australia
}

\begin{abstract}
This paper discusses how high spatial and spectral resolution observations of bow-shock 'bullets' in Orion are challenging our understanding of molecular shock physics.
\end{abstract}

\section{Introduction}

The Orion Nebula is our nearest region of massive star formation, the site of the complete range of phenomena associated with star formation and early stellar evolution. Amongst these is the shock-excitation of molecular gas, the result of mass-ejection from one or more of the luminous IR sources in the core of the molecular cloud OMC-1. It is easily the brightest $\mathrm{H}_{2}$ emission source available for study (Beckwith et al., 1978). However, the structure of the $\mathrm{H}_{2}$ emission nebula has only become clear recently, with the advent of both high spatial resolution and large area coverage imaging. While it has a broadly bipolar morphology, in addition there is also a set of 'fingers' pointing away from the centre of the cloud. The tips of the most distant fingers emit strongly in the near-IR lines of [FeII], as well as in optical lines such as [OI] and [SII] (Allen \& Burton 1993, Axon \& Taylor 1984). It appears that each finger is a bow-shock, a 'bullet'-like head followed by a lower-excitation $\mathrm{H}_{2}$-emitting wake. These bullets are typically $2-3$ " in size, just resolvable from the ground, but with HST we are now able to dissect their structure.

\section{Anatomy of a Bow Shock}

While most detailed modeling of the emission from molecular shocks has dealt with planar shock fronts, it is clear that in general more complicated geometries exist and observations of shocks thus sample a range of environmental conditions. This is illustrated in Figure 1, a schematic of an interstellar bullet interacting with ambient gas. A bow wake is formed around the bullet, with the local shock speed varying as the cosine of the direction to the normal. At the head, where the speeds are highest, the molecular gas is dissociated. Emission comes from a cooling region along the bow, whose thickness depends on the mode of excitation (i.e., whether the shock is $\mathrm{C}$-type or J-type?), and for a particular species the intensity depends on the local shock speed to a power of $\sim 1-3$. The excitation falls along the wake until the gas is no longer shocked. The bullet is inclined to our line of sight, and an observer resolves a section of the bow which thus includes a range of excitation conditions. 
Further complicating the issue, in addition to whether the shock is $\mathrm{C}-$ or $\mathrm{J}$-type, is the possible existence of a pre-cursor which would excite $\mathrm{H}_{2}$ upstream of higher-excitation optical emission. The ambient gas will also be entrained; this may be by steady entrainment along the wake, or by prompt entrainment at the bullet's head where the material is simply pushed aside by its passage. As the gas cools in the wake, instabilities are expected to occur. The cooling length of a $\mathrm{C}$-shock is typically a few $\times 10^{15} \mathrm{~cm}\left(\times 0.14^{\prime \prime}\right.$ at the distance of Orion, e.g., Draine et al., 1983), and thus resolvable with HST.

\section{Observations}

Both the wide profiles of the $\mathrm{H}_{2}$ lines and thermal nature of its emission spectrum indicate that the $\mathrm{H}_{2}$ is shock-excited (e.g., Nadeau \& Geballe 1979, Brand et al., 1988, Moorhouse et al., 1990) in Orion. The bullets themselves display classical low-excitation $\mathrm{HH}-$ object spectra in the optical and near-IR (e.g., Axon \& Taylor 1984, Burton \& Allen 1994). Their $\sim 300 \mathrm{~km} / \mathrm{s}$ wide, slightly assymetric line profiles (Tedds et al., 1995) is as expected from the integrated emission of bow-shocks with a range of inclination angles to our line of sight (Hartigan et al., 1987). Recent high spatial and spectral resolution $\mathrm{H}_{2}$ line profiles exhibit localised regions of line splitting, a few arcsec in extent, interior to the region of the optical bullets (Chrysostomou et al., 1997). This has been interpreted as evidence for a population $\mathrm{H}_{2}$-only bow-shocks in the core, in addition to the widespread $\mathrm{H}_{2}$ emission excited by the interaction of a steady wind or outflow with the ambient medium.

We (O'Dell, Hartigan \& Burton, 1997) have recently observed the optical bullets in the emission lines of [OI], [SII], $\mathrm{H} \alpha$ and [OIII] with HST at 0.1 " spatial resolution $\left(\equiv 7 \times 10^{14} \mathrm{~cm}\right)$, tracers of a range of excitation conditions. In general, there are clear excitation gradients from head to tail along the bullets; [OIII] or $\mathrm{H} \alpha$ emission from the head, then $\mathrm{H} \alpha$ or [SII] along its outer edge, and [SII] in its tail. The [SII] essentially displays the same distribution as the near-IR [FeII], albeit seen at much higher resolution. The emission then fades into $\mathrm{H}_{2}$ in the wake. A typical example is shown in Fig. 2 for the knot $\mathrm{HH}-207$.

In practice, however, each knot displays different specific characteristics. Compact emission knots are visible in many, as are lower-excitation knots upstream of the head. The influence of the foreground HII region is evident. In the knots in the southern portion of the nebula, particularly to the south of the Trapezium, external photo-ionization is apparent through enhanced emission of [OIII] along one side of the knot. In the bullets to the NW of OMC-1, on the other hand, ionization gradients exist across the field but appear to be associated with simply foreground emission from the $\mathrm{HII}$ region; the $\mathrm{HH}$-knots are shielded from direct UV illumination.

\section{Interpretation}

There are clear signs of excitation gradients along the bullets as would be expected in bow-shocks, but also many other features which will necessitate specific modeling to interpret. Are the knots of lower-excitation emission upstream of higher-excitation knots indeed caused by a pre-cursor? Are the knots along 


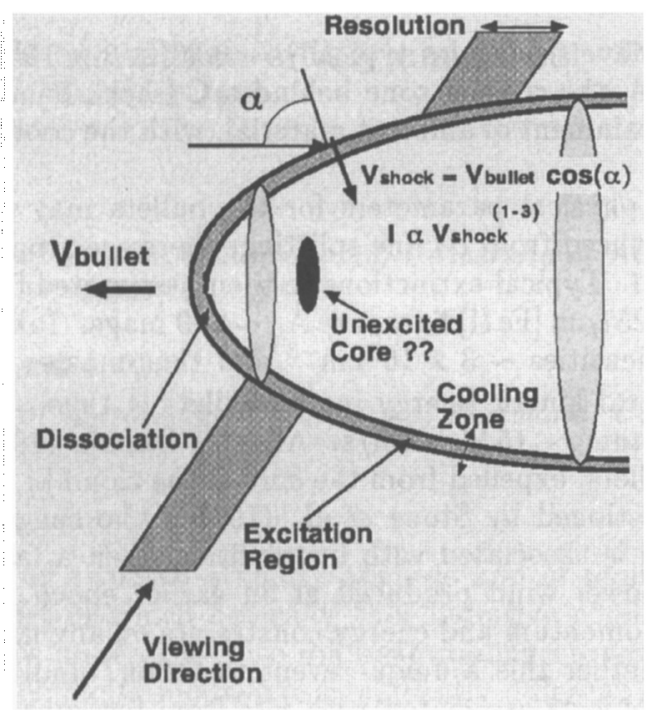

Figure 1. Schematic representation of a bow shock around a bullet. Line emission occurs in the shaded cooling zone along the sides of the bow, with the intensity related to the normal component of the bullet velocity, which decreases along the wake. Gas in the cap is dissociated. The bow is at angle to the line of sight, with the observer's spatial resolution indicated by the shaded band.
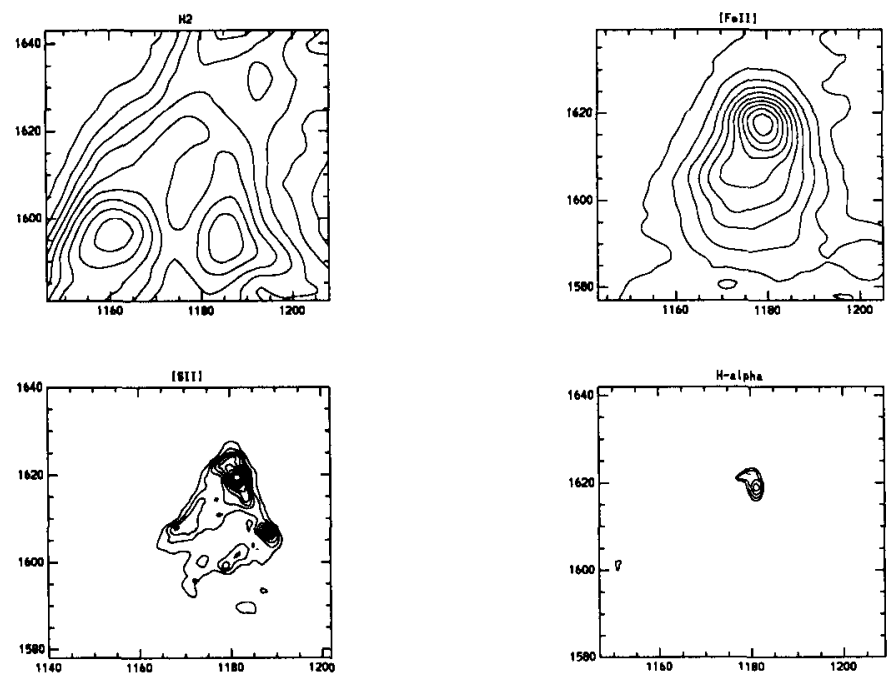

Figure 2. Contour maps of the HH-207 bow shock. From top-left, in a clockwise direction, are shown $\mathrm{H}_{2}(2.122 \mu \mathrm{m})$, [Fe II] $(1.644 \mu \mathrm{m}), \mathrm{H} \alpha$ $(6563 \AA)$ and $[\mathrm{SII}](6717 / 31 \AA)$. The first two are from AAT observations and the last two from HST. 20 pixels $\equiv 2^{\prime \prime} \equiv 1.4 \times 10^{16} \mathrm{~cm}$, at $480 \mathrm{pc}$. 
the wakes, which have size scales typically $\sim 0.3^{\prime \prime}\left(\equiv 2 \times 10^{15} \mathrm{~cm}\right)$ indicative of cooling instabilities, the cooling zone behind a $\mathrm{C}$-shock being resolved? Or do they represent entrainment of ambient material, with the cooling zone remaining unresolved?

A number of physical parameters for the bullets may now be estimated. Including those deduced from $\mathrm{H}_{2}$ line splitting, there are around 50 bullets associated with $\mathrm{OMC}-1$. Typical extinctions to them, estimated from measurements of the 1.644 and $1.257 \mu \mathrm{m}$ [Fe II] lines, are $\mathrm{A}_{v} \sim 4-9$ mags. Taken with their sizes, it suggests mean densities $\sim 3 \times 10^{5} \mathrm{~cm}^{-3}$ and thus masses of order $10^{-3} \mathrm{M}_{\odot}$ per bullet. The total kinetic energy in the bullets is thus $\sim 5 \times 10^{46}$ ergs and their total momentum $\sim 15 \mathrm{M} \odot \mathrm{km} / \mathrm{s}$. Allen \& Burton (1993) proposed that they are indeed bullets, expelled from the core of the cloud in an explosive event. This has been questioned by Stone et al. (1995), who suggest they are dense knots whose origin is associated with instabilities when a fast, impulsive wind interacts with a slower wind produced at an earlier epoch. The observations place prodigious momentum and energy constraints on any model. There is also the question of whether this a unique event to Orion? Individually, the knots resemble the $\mathrm{HH}$-objects associated with jets from low-mass stars. Taken collectively, however, they clearly have a different origin, possibly connected with several of the phenomena occurring in a high-mass star forming region.

Acknowledgments. This work would not have been possible with the substantial contributions of many colleagues. More complete treatments of it will appear with different lead authors. I particularly wish to thank Peter Brand, Antonio Chrysostomou, Pat Hartigan and Bob O'Dell for their efforts.

\section{References}

Allen, D. \& Burton, M. 1993, Nature, 363, 54

Axon, D. \& Taylor, K. 1984, MNRAS, 207, 241

Beckwith, S., Persson, S., Neugebauer, G. \& Becklin, E. 1978, ApJ, 223, 464

Brand, P, Moorhouse, A., Burton, M., Geballe, T., Bird, M. \& Wade, R. 1988, ApJ, 334, L103

Burton, M. \& and Allen, D. 1994, Astrophysics and Space Science Library, 190, 61 (Kluwer)

Chrysostomou, A., Burton, M., Axon, D, Brand, P., Hough, J, Bland-Hawthorn, J \& Geballe, T. 1997, MNRAS, submitted

Draine, B., Roberge, W. \& Dalgarno, A. 1983. ApJ, 264, 485

Hartigan, P., Raymond, J. \& Hartmann, L. 1987, ApJ, 316, 323

Moorhouse, A., Brand, P., Geballe, T. \& Burton, M. 1990, MNRAS, 242, 88

Nadeau, D. \& Geballe, T. 1979, ApJ, 230, 169

O'Dell, C., Hartigan, P. \& Burton, M. 1997, in preparation

Stone, J., Xu, J. \& Mundy, L. 1995, Nature, 377, 315

Tedds, J., Brand, P., Burton, M., Chrysostomou, A. \& Fernandes, A. 1995, Ap\&SS, 224, 139 